\title{
Investigation of Melioidosis Using Blood Culture and Indirect Hemagglutination Assay Serology among Patients with Fever, Northern Tanzania
}

Michael J. Maze, ${ }^{1,2,3 \star}$ Mindy Glass Elrod, ${ }^{4}$ Holly M. Biggs, ${ }^{5,6}$ John Bonnewell,,${ }^{5,6}$ Manuela Carugati, ${ }^{6}$ Alex R. Hoffmaster, ${ }^{4}$ Bingileki F. Lwezaula, ${ }^{7}$ Deng B. Madut, ${ }^{5,6}$ Venance P. Maro, ${ }^{3,10}$ Blandina T. Mmbaga, ${ }^{3,9,10}$ Anne B. Morrissey, ${ }^{5,6}$ Wilbrod Saganda, ${ }^{7}$ Philoteus Sakasaka, ${ }^{9}$ Matthew P. Rubach, ${ }^{5,6,8}$ and John A. Crump ${ }^{2,5,6,7,10}$

${ }^{1}$ Department of Medicine, University of Otago, Christchurch, New Zealand; ${ }^{2}$ Centre for International Health, University of Otago, Dunedin, New Zealand; ${ }^{3}$ Kilimanjaro Christian Medical Centre, Moshi, Tanzania; ${ }^{4}$ Bacterial Special Pathogens Branch, US Centers for Disease Control, Atlanta, Georgia; ${ }^{5}$ Duke Global Health Institute, Duke University, Durham, North Carolina; ${ }^{6}$ Division of Infectious Diseases and International Health, Department of Medicine, Duke University Health System, Durham, North Carolina; ${ }^{7}$ Mawenzi Regional Referral Hospital, Moshi, Tanzania; ${ }^{8}$ Programme in Emerging Infectious Diseases, Duke-National University of Singapore, Singapore, Singapore; ${ }^{9}$ Kilimanjaro Clinical Research Institute, Moshi, Tanzania; ${ }^{10}$ Kilimanjaro Christian Medical University College, Tumaini University, Moshi, Tanzania

\begin{abstract}
Prediction models indicate that melioidosis may be common in parts of East Africa, but there are few empiric data. We evaluated the prevalence of melioidosis among patients presenting with fever to hospitals in Tanzania. Patients with fever were enrolled at two referral hospitals in Moshi, Tanzania, during 2007-2008, 2012-2014, and 2016-2019. Blood was collected from participants for aerobic culture. Bloodstream isolates were identified by conventional biochemical methods. Non-glucose-fermenting Gram-negative bacilli were further tested using a Burkholderia pseudomallei latex agglutination assay. Also, we performed $B$. pseudomallei indirect hemagglutination assay (IHA) serology on serum samples from participants enrolled from 2012 to 2014 and considered at high epidemiologic risk of melioidosis on the basis of admission within 30 days of rainfall. We defined confirmed melioidosis as isolation of $B$. pseudomallei from blood culture, probable melioidosis as a $\geq 4$-fold rise in antibody titers between acute and convalescent sera, and seropositivity as a single antibody titer $\geq 40$. We enrolled 3,716 participants and isolated non-enteric Gram-negative bacilli in five (2.5\%) of 200 with bacteremia. As none of these five isolates was $B$. pseudomallei, there were no confirmed melioidosis cases. Of 323 participants tested by IHA, $142(44.0 \%)$ were male, and the median (range) age was $27(0-70)$ years. We identified two $(0.6 \%)$ cases of probable melioidosis, and 57 (17.7\%) were seropositive. The absence of confirmed melioidosis from 9 years of fever surveillance indicates melioidosis was not a major cause of illness.
\end{abstract}

\section{INTRODUCTION}

The importance of Burkholderia pseudomallei, the agent of melioidosis, as a cause of illness in Africa is yet to be fully determined. There have been occasional reports of individual cases and a study indicating that melioidosis is present in coastal Kenya. $^{1-4}$ To our knowledge, there are no published data from Tanzania to indicate whether melioidosis is present, ${ }^{5}$ but prediction models based on environmental suitability and populations at risk suggest that $B$. pseudomallei may be endemic in Tanzania. ${ }^{6}$

Burkholderia pseudomallei infection most commonly results in subclinical or self-limiting symptoms in immunocompetent individuals. ${ }^{7}$ Acute symptomatic infection with $B$. pseudomallei typically presents with bacteremia, pneumonia, and localized abscesses. Burkholderia pseudomallei can be cultured from blood in only a proportion of patients with melioidosis, as bacteremia is thought to occur in $40-60 \%,{ }^{7}$ with other patients having localized disease. In diagnostic accuracy studies using latent class models, blood culture has an estimated sensitivity of approximately $60 \%$ for melioidosis. $^{8}$ In culture on solid media, B. pseudomallei can be overlooked as a contaminant, as colonies appear morphologically similar to common contaminants such as Pseudomonas stutzeri. ${ }^{9}$ Finally, B. pseudomallei can be misidentified as another Gram-negative organism such as Burkholderia cepacia or Chromobacterium violaceum by routinely used identification systems, ${ }^{10,11}$ and it is not included in standard matrix-assisted laser desorption ionization-time of flight mass

*Address correspondence to Michael J. Maze, Department of Medicine, University of Otago Christchurch, 2 Riccarton Rd., Christchurch 8011, New Zealand. E-mail: michael.maze@otago.ac.nz spectrometry databases. ${ }^{12}$ As such, melioidosis may be underappreciated or not recognized through routine diagnostic testing in areas where it is uncommon, or the prevalence is unknown.

Burkholderia pseudomallei indirect hemagglutination assay (IHA) is a widely used serologic test for melioidosis. ${ }^{9}$ Although use of IHA for the diagnosis of acute melioidosis is limited in endemic countries because of background seropositivity, it remains useful for determining exposure to $B$. pseudomallei. ${ }^{9}$ Population seropositivity in turn informs our understanding of the population-level risk of melioidosis. We sought to understand the prevalence of melioidosis and exposure in northern Tanzania through systematic blood culture testing among patients hospitalized with fever, and IHA serology among a high-risk subset of patients.

\section{METHODS}

Study setting. We conducted prospective hospital-based fever surveillance studies at Kilimanjaro Christian Medical Centre (KCMC) and Mawenzi Regional Referral Hospital (MRRH) in Moshi, Tanzania, during the periods of September 17, 2007 through August 31, 2008, September 26, 2011 through May 31, 2014, and September 6, 2016 through May 31, 2019. Kilimanjaro Christian Medical Centre is a 630-bed zonal referral, and MRRH is a 300-bed regional hospital. Moshi (population $>180,000$ ) is the administrative center of the Kilimanjaro region (population $>1.6$ million) and is situated at an elevation of approximately $890 \mathrm{~m}$ above sea level. The climate is tropical, with rainy seasons from March through May and October through December. Agriculture in northern Tanzania includes smallholder systems involving mixed crop and 
livestock farming, as well as pastoralism. Continuously irrigated rice farming, an established risk factor for melioidosis, is increasingly practiced by farmers within the area served by our two sentinel site hospitals. ${ }^{13}$

Study procedures. We prospectively enrolled pediatric and adult inpatients at KCMC and MRRH during each time period, and during the 2012-2014 time period, we also enrolled outpatients. Adolescents and adults, defined as age $\geq 13$ years, were eligible to participate if they had an oral temperature of $\geq 38.0^{\circ} \mathrm{C}$, or, during $2012-2014$, a history of fever within the previous 72 hours. Infants and children, defined as age $\geq 2$ months to $<13$ years, were eligible to participate if they had a history of fever in the past 48 hours, an axillary temperature of $\geq 37.5^{\circ} \mathrm{C}$, or a rectal temperature of $\geq 38.0^{\circ} \mathrm{C}$. We enrolled consecutive eligible inpatients and, during 2012-2014, every second eligible outpatient. Outpatients were included as the primary study sought to describe the incidence of bacterial zoonoses and bloodstream infections, which included diseases that might present to and be managed in the outpatient setting, such as typhoid fever. ${ }^{14,15}$ All patients were enrolled within 24 hours of admission. After obtaining informed consent, a trained study team member completed standardized case report forms and drew blood for culture and serologic testing. The case report form varied during each time period but included demographic details, symptoms, and use of antibacterial drugs before enrollment. Information regarding rainfall in the 30 days preceding enrollment was obtained for the 2012-2014 period from the Tanzanian production company rainfall station near Moshi.

Blood culture. We drew blood for a single aerobic blood culture from participants at enrollment, which was loaded into the BacT/ALERT 3D microbial detection system (BioMérieux, Marcy-l'Étoile, France), and incubated for up to 5 days. We inoculated $4 \mathrm{~mL}$ of blood into BacT/ALERT pediatric FAN aerobic bottles (2007-2008 and 2012-2014) or pediatric PF plus bottles (2016-2019) (BioMérieux) for pediatric participants (aged $<13$ years) and $10 \mathrm{~mL}$ of blood into BacT/ALERT standard aerobic bottles (2007-2008 and 2012-2014) or FA plus bottles (2016-2019) (BioMérieux) for adult participants (aged $\geq 13$ years). Blood cultures were assessed for volume adequacy by measuring the weight before and after inoculation. Adequate blood volume was defined as $\pm 20 \%$ of the target blood volume. Bloodstream isolates were identified by conventional methods: following testing on the API20NE (BioMérieux) biochemical identification system, non-glucosefermenting Gram-negative bacilli were further tested by B. pseudomallei latex agglutination (Mahidol University, Bankok, Thailand) test. ${ }^{9,16}$

Serology testing. During the 2012-2014 study, we collected blood for acute serum at enrollment and convalescent serum 4-6 weeks later. Blood was allowed to clot for between 30 and 60 minutes. It was then centrifuged for 15 minutes at $1,126-1,455$ relative centrifugal force to separate serum. Serum was stored at $-80^{\circ} \mathrm{C}$. Serum specimens were batch shipped on dry ice from Moshi, Tanzania, to Atlanta, GA, for testing. Serology testing was performed at the U.S. CDC. The convalescent serum of a subset of participants considered at high epidemiologic risk of melioidosis on the basis of admission within 30 days of rainfall was tested using $B$. pseudomallei IHA. We performed $B$. pseudomallei IHA on paired acute and convalescent sera of participants with a reciprocal titer $\geq 40$ on their convalescent serum. We performed B. pseudomallei IHA using antigen pooled from two clinical B. pseudomallei isolates from Southeast Asia and Australia following standard U.S. CDC laboratory protocols. ${ }^{17}$

Case definitions. A confirmed case of melioidosis was defined as isolation of $B$. pseudomallei from blood culture, probable melioidosis as $a \geq 4$-fold rise in antibody titers between acute and convalescent sera, and seropositivity as a single reciprocal antibody titer $\geq 40$. $^{18,19}$

Statistical analysis. Case report form and laboratory data were entered using the Cardiff Teleform system (OpenText, Waterloo, Canada) into an Access database (Microsoft Corporation, Redmond, WA). Descriptive analyses, including median and range for continuous variables and proportions for categorical variables, were performed using Stata, version 16.0 (StataCorp, College Station, TX).

Research ethics. This study was conducted in accordance with the Declaration of Helsinki. It was approved by the KCMC Research Ethics Committee (\#295), the Tanzania National Institute for Medical Research National Ethics Coordinating Committee (NIMR1HQ/R.8cNo1. 11/283), Duke University Medical Center Institutional Review Board (IRB\#Pro00016134), and the University of Otago Human Ethics Committee (Health) (H15/055). Written informed consent was obtained from all participants or their guardians.

\section{RESULTS}

We enrolled 3,716 participants who had blood culture performed. Participant characteristics are shown in Table 1. Of note, the median (range) age was $20(<1,84)$ years. The median (range) duration of illness was $4(1,120)$ days. Of 1,752 participants for whom corresponding rainfall data were available, there were $1,472(84.0 \%)$ who presented within 30 days of rain.

Among those with data available, 2,658 (73.1\%) of 3,637 participants had adequately filled blood culture bottles. Among pediatric participants, 734 (51.4\%) of 1,427 blood culture bottles were adequately filled, and among adults 1,924 $(87.1 \%)$ of 2,210 , blood culture bottles were adequately filled. Of responding participants, 849 (32.6\%) of 2,607 reported taking antibacterial drugs before enrollment. Non-enteric

TABLE 1

Characteristics of participants undergoing blood culture, Tanzania, 2007-2019 $(N=3,716)$

\begin{tabular}{lcc}
\hline & $N$ & $n(\%)$ \\
\hline $\begin{array}{l}\text { Demographic characteristics } \\
\text { Age (years), median (IQR) }\end{array}$ & 3,573 & $20.3(2.0-39.3)$ \\
$\quad$ Gender, male & 3,668 & $1,833(50.0)$ \\
Risk factors & & \\
$\quad$ Rainfall in 30 days before admission & 1,738 & $24.0(1.8-67.7)$ \\
$\quad$ (mm), median (IQR) & & \\
$\quad$ Farming occupation & 1,445 & $261(18.1)$ \\
$\quad$ Worked in rice field & 1,445 & $23(1.6)$ \\
$\quad$ Self-reported HIV-infected or & 2,052 & $656(32.0)$ \\
$\quad$ positive HIV serology & & \\
Clinical history & & \\
$\quad$ IIIness duration (days), median (IQR) & 3,334 & $4(3-9)$ \\
$\quad$ Cough & 3,234 & $1,936(59.9)$ \\
$\quad$ Dyspnea & 3,227 & $2,216(31.3)$ \\
$\quad$ Headache & 2,480 & $1,551(62.5)$ \\
$\quad$ Myalgia & 2,371 & $947(40.0)$ \\
Rash or cutaneous lesion & 2,076 & $158(7.6)$ \\
\hline IQR = interquartile range; $N=$ number of participants with data available.
\end{tabular}


Gram-negative bacilli were isolated from the blood culture of five $(0.1 \%)$ participants. None was determined to be $B$. pseudomallei, so no cases of confirmed melioidosis were identified.

Among 323 participants tested by IHA, the median (range) age was $27(0-70)$ years, and the median (range) duration of fever was 4 (1-60) days. Other characteristics of participants tested by IHA are shown in Table 2. Forty-four (13.6\%) reported being HIV infected. Two $(0.6 \%)$ cases of probable melioidosis were identified through paired serology, both of whom survived until follow-up. Neither reported recent travel outside Kilimanjaro region. The first case of probable melioidosis was a 6-year-old boy with a 2-day history of fever, rigors, cough, and dyspnea. The participant and his guardians reported a previous negative HIV test. The presence of other immune-suppressing diseases, including diabetes mellitus, ${ }^{7}$ or medications was not ascertained. He reported exposure to standing water around his house and walking barefoot in the mud during the preceding 30 days. A discharge diagnosis of pneumonia was recorded by the treating clinical team. The patient was treated with oral azithromycin. The second case of probable melioidosis was a 36-year-old woman with a 3-day history of fever, rigors, cough, and joint pain. She reported a previous negative HIV test. The presence of other immunesuppressing diseases or medications was not ascertained. She reported no exposure to surface or standing water around her house, and she did not report walking barefoot in the preceding 30 days. She was treated with analgesia and without antimicrobials. A discharge diagnosis of rheumatoid arthritis was recorded by the clinical team.

We identified 57 (17.7\%) participants who met the definition for $B$. pseudomallei seropositivity. The highest reciprocal antibody titer was 10,240 (Figure 1). Among participants who were seropositive, $24(42.1 \%)$ were male, and the median (range) age was $23(<1,84)$ years. Of seropositive participants, $14(24.6 \%)$ reported exposure to surface water compared with $50(18.8 \%)$ seronegative participants $(P=0.32), 19$ $(33.3 \%)$ reported the presence of standing water around their house compared with 71 (26.8\%) of seronegative participants
$(P=0.32)$, and $26(44.6 \%)$ reported walking barefoot in the preceding 30 days compared with $118(44.4 \%)$ of seronegative participants $(P=0.86)$.

\section{DISCUSSION}

This study did not detect a confirmed case of melioidosis in northern Tanzania despite nearly a decade of blood culture in more than 3,700 patients hospitalized with fever. Melioidosis was unlikely to be a major cause of severe febrile illness in northern Tanzania during the study period. Across three study surveillance periods, we cultured blood of consecutive patients hospitalized with fever and systematically investigated isolates to identify $B$. pseudomallei. The absence of $B$. pseudomallei blood isolates among our patient population does not preclude the occurrence of melioidosis in our region. In Kilifi, Kenya, four $B$. pseudomallei isolates were recovered from $>66,000$ blood cultures to provide an estimated annual incidence of 0.2 cases per 100,000 people. ${ }^{4}$ Nonetheless, our findings would indicate that if present melioidosis is likely to be a rare cause of bacteremia in northern Tanzania.

Two participants met the case definition for probable melioidosis. Both cases had negative blood cultures but did not have sputum or other potentially relevant samples cultured. Although each probable case reported a clinical illness that was compatible with melioidosis, the clinical features were nonspecific, and both survived the acute illness without receiving antimicrobials active against $B$. pseudomallei. ${ }^{7}$ Furthermore, we did not ascertain whether either patient had host risk factors for melioidosis such as diabetes or immune suppression. As IHA has limitations in the diagnosis of melioidosis and is not recommended as a diagnostic test in endemic areas, ${ }^{9}$ it is uncertain whether their illness was due to melioidosis.

Almost $20 \%$ of participants who underwent IHA testing in our study were seropositive to $B$. pseudomallei. Whether IHA seropositivity indicates exposure to $B$. pseudomallei or an antigenically similar organism such as Burkholderia mallei or another Burkholderia spp. is uncertain. ${ }^{20}$ To our knowledge,

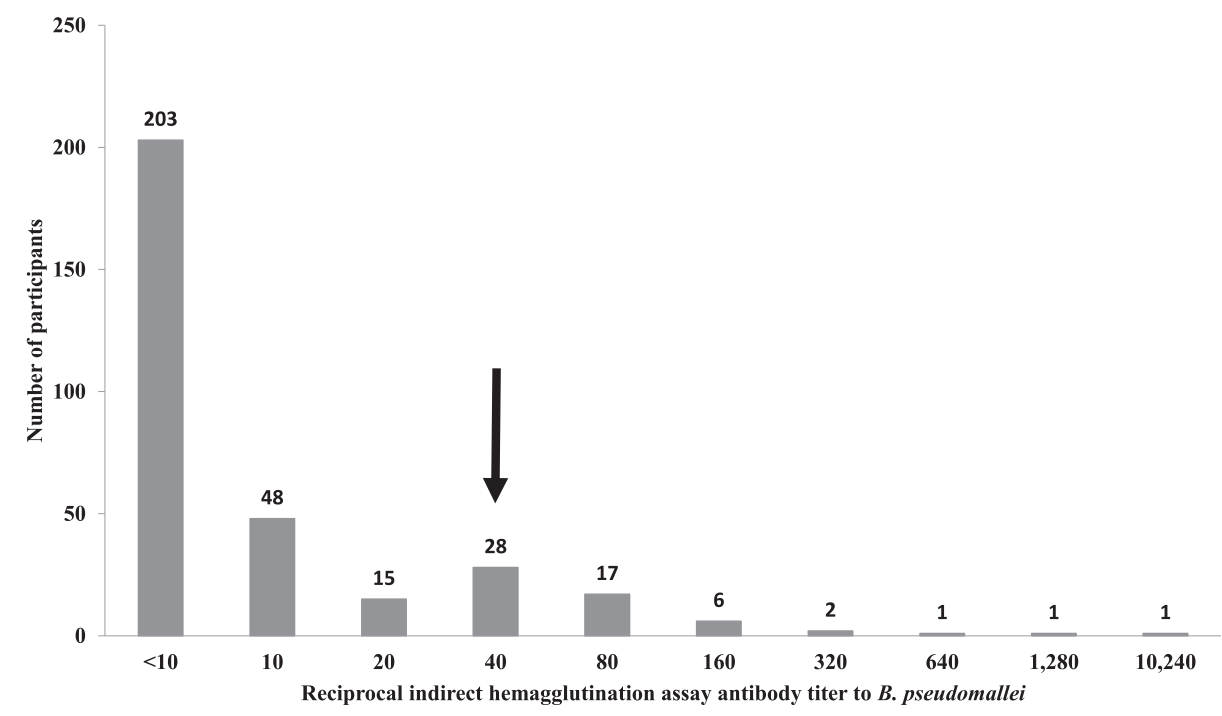

FIGURE 1. Distribution of Burkholderia pseudomallei indirect hemagglutination test reciprocal antibody titers among febrile patients, Tanzania, 2012-2014. Key: Arrow at a reciprocal titer of 40 indicates cutoff for seropositivity. 
TABLE 2

Characteristics of participants undergoing Burkholderia pseudomallei indirect hemagglutination testing, Tanzania, 2012-2014 $(N=323)$

\begin{tabular}{lc}
\hline & $n(\%)$ \\
\hline Demographic characteristics & \\
Age (years), median (IQR) & $27(5-40)$ \\
Gender, male & $142(44.0)$ \\
Risk factors & $46(27-72)$ \\
$\quad$ Rainfall in 30 days before admission (mm), & \\
$\quad$ median (IQR) & $66(20.4)$ \\
Farming occupation & $44(13.6)$ \\
Self-reported HIV-infected & \\
Clinical history & $4(2-7)$ \\
Fever duration (days), median (IQR) & $200(61.9)$ \\
Cough & $101(31.3)$ \\
Dyspnea & $217(67.2)$ \\
Headache & $140(43.3)$ \\
Myalgia & $24(7.4)$ \\
Rash or cutaneous lesion &
\end{tabular}

there have not been data published on $B$. pseudomallei seropositivity in Tanzania. ${ }^{21}$ In Uganda, a study by Frazer ${ }^{22}$ identified $25(5.9 \%)$ of 426 individuals as seropositive to $B$. pseudomallei using IHA. The Frazer study surveyed healthy adults from the capital, Kampala, as well as rural areas, and most seropositive participants had reciprocal antibody titers $\leq 160$. The relatively high seroprevalence and the absence of bacteremia seen in our study are notable. The seroprevalence in our study was higher than a recent report from Australia, ${ }^{23}$ but lower than a study in Thailand. ${ }^{24}$ Both countries are highly endemic for melioidosis, with annual incidence estimates in northern Australia of up to 50 cases per 100,000 people. ${ }^{25}$ In Thailand, it is considered that 1 in 4,600 antibody-producing exposures result in clinically apparent melioidosis. ${ }^{24} \mathrm{~A}$ smaller ratio (i.e., fewer exposures per clinically apparent case) is estimated for Australia. ${ }^{23}$ Although exposure to B. pseudomallei is a plausible cause of seropositivity, exposure to antigenically similar organisms, such as $B$. mallei and Burkholderia thailandensis, is also possible. ${ }^{20,26}$ Burkholderia mallei, the agent that causes glanders, serologically cross-reacts with $B$. pseudomallei but is rarely identified in Tanzania. ${ }^{20,27}$ Burkholderia thailandensis is a nonpathogenic species that is not known to be present in Tanzania, but has been identified in West Africa. ${ }^{28}$ Further work is needed before it can be determined whether or not melioidosis is endemic in northern Tanzania. Soil sampling using established guidelines to determine whether $B$. pseudomallei or an antigenically similar bacterium is present would be a useful strategy in addition to continued clinical surveillance. ${ }^{29}$

Our study determined that many participants had exposure to surface water and undertook activities that might place them at risk for melioidosis. However, we had selected participants for testing if they had presented within 30 days of rainfall, as recent rainfall is an established risk factor for melioidosis in endemic countries. The strategy of testing only participants with known risk factors introduced bias, which precluded a formal risk factor analysis.

Although our study used a systematic approach to evaluating blood culture isolates, limitations in the methodology may underestimate the prevalence of melioidosis. In particular, not all patients with fever were enrolled; some participants had inadequately filled blood culture bottles and some participants reported having taken antibacterial drugs before enrollment. ${ }^{30}$ In addition, culture solely of blood for $B$. pseudomallei will have underestimated the prevalence, and systematic culture of urine, sputum, pus and throat swabs is likely to have increased diagnosis. The $B$. pseudomallei seroprevalence cannot be considered a true community prevalence because testing was targeted toward a subset of patients.

In conclusion, we have not identified culture-confirmed melioidosis despite nearly a decade of careful surveillance. Based on these data, we would not recommend that clinicians in northern Tanzania treat febrile patients with antimicrobials that cover melioidosis empirically. However, given the IHA results, it remains possible that melioidosis is present in northern Tanzania and an index of suspicion of melioidosis among patients with fever, and culture of blood and multiple clinical specimen types remain critical to ensure that persons with melioidosis are appropriately identified and treated.

Received March 1, 2020. Accepted for publication August 4, 2020.

Published online September 28, 2020.

Acknowledgments: We would like to thank those involved in recruitment, laboratory work, data management, and study administration, including Godfrey S. Mushi, Flora W. Mboya, Lilian E. Ngowi, Winfrida H. Shirima, Michael E. Butoyi, Anna H. Mwalla, Miriam L. Barabara, Ephrasia Mariki, Edna Ngowi, Olterere Salimu, Rither Mhela, Jamal Bashiri, Christopher Swai, Jerome Mlangi, Tumsifu G. Tarimo, Yusuf S. Msuya, Leila J. Sawe, Aaron E. Tesha, Luig J. Mbuya, Edward M. Singo, Stephen Sikumbili, Erica Chuwa, Daniel Mauya, Isaac A. Afwamba, Thomas M. Walongo, Remigi P. Swai, Augustine M. Musyoka, Rose Oisso, Gershom Mmbwambo, Philoteus A. Sakasaka, O. Michael Omondi, Enoch J. Kessy, Alphonse S. Mushi, Robert S. Chuwa, Charles Muiruri, Cynthia A. Asiyo, Frank M. Kimaro, and Francis $P$. Karia. In addition, we would like to thank the study participants as well as the clinical staff and administration at Kilimanjaro Christian Medical Centre and Mawenzi Regional Referral Hospital for their support during this study.

Financial support: This research was supported by the joint U.S. National Institutes of Health (NIH: www.nih.gov)-Science Foundation (NSF: www.nsf.gov) Ecology of Infectious Disease program (R01TW009237) and the Research Councils UK, Department for International Development (UK) and UK Biotechnology and Biological Sciences Research Council (BBSRC: www.bbsrc.ac.uk) (grant numbers BB/J010367/1, BB/L018926, BB/L017679, and BB/L018845), U.S. NIH awards (U01 Al062563 and R01 Al121378); and the Bill \& Melinda Gates Foundation funded Typhoid Fever Surveillance in subSaharan Africa Program (TSAP) grant (OPPGH5231). M. J. M. received support from University of Otago scholarships: the Frances G. Cotter Scholarship and the McGibbon Travel Fellowship. H. M. B. received support from the National Institutes of Health Interdisciplinary Research Training Program in AIDS (grant number NIAID-AI007392). D. B. M. received support from the NIH Ruth L. Kirschstein National Research Service award (NRSA) 5T32AI007392 and U.S. NIH Fogarty International Center grant D43TW009337. M. P. R. received support from the National Institutes of Health Research Training grants (R25 TW009337 and R25 TW009343) funded by the Fogarty International Center and the National Institute of Mental Health and from U.S. NIH K23 Al116869.

Disclaimer: The findings and conclusions in this article are those of the authors and do not necessarily represent the official position of the U.S. CDC.

Authors' addresses: Michael J. Maze, Mindy Glass Elrod, and Alex R. Hoffmaster, US Centers for Disease Control and Prevention, Atlanta, GA, E-mails: michael.maze@otago.ac.nz, wzg0@cdc.gov, and amh9@cdc.gov. Holly M. Biggs, John Bonnewell, Manuela Carugati, Deng B. Madut, Anne B. Morrissey, and Matthew P. Rubach, Duke University, Durham, NC, E-mails: hollybiggs1@gmail.com, john. bonnewell@duke.edu, manuela.carugati@duke.edu, deng.madut@ duke.edu, amorr1023@gmail.com, and matthew.rubach@duke.edu. Bingileki F. Lwezaula and Wilbrod Saganda, Mawenzi Regional 
Referral Hospital, Moshi, Tanzania, E-mail: Iwezaula@gmail.com and. Venance P. Maro, Blandina T. Mmbaga, and Philoteus Sakasaka, Kilimanjaro Clinical Research Institute, Moshi, Tanzania, E-mails: venmaro@ ymail.com, blaymt@gmail.com, and psakasaka@yahoo.com. John A. Crump, University of Otago, Dunedin, New Zealand, E-mail: john.crump@ otago.ac.nz.

This is an open-access article distributed under the terms of the Creative Commons Attribution (CC-BY) License, which permits unrestricted use, distribution, and reproduction in any medium, provided the original author and source are credited.

\section{REFERENCES}

1. Dan $M, 2015$. Melioidosis in travelers: review of the literature. J Travel Med 22: 410-414.

2. Katangwe T, Purcell J, Bar-Zeev N, Denis B, Montgomery J, Alaerts M, Heyderman RS, Dance DA, Kennedy N, Feasey N, 2013. Human melioidosis, Malawi, 2011. Emerg Infect Dis 19: 981-984.

3. Morosini Ml, Quereda C, Gil H, Anda P, Nunez-Murga M, Canton R, Lopez-Velez R, 2013. Melioidosis in traveler from Africa to Spain. Emerg Infect Dis 19: 1656-1659.

4. Muthumbi EM et al., 2019. Population-based estimate of melioidosis, Kenya. Emerg Infect Dis 25: 984-987.

5. Marchello CS, Dale AP, Pisharody S, Rubach MP, Crump JA, 2019. A systematic review and meta-analysis of the prevalence of community-onset bloodstream infections among hospitalized patients in Africa and Asia. Antimicrob Agents Chemother 64: e01974-19.

6. Limmathurotsakul D et al., 2016. Predicted global distribution of Burkholderia pseudomallei and burden of melioidosis. Nat Microbiol 1: 15008.

7. Wiersinga WJ, Virk HS, Torres AG, Currie BJ, Peacock SJ, Dance DA, Limmathurotsakul D, 2018. Melioidosis. Nat Rev Dis Primers 4: 17107.

8. Limmathurotsakul D et al., 2010. Defining the true sensitivity of culture for the diagnosis of melioidosis using Bayesian latent class models. PLoS One 5: e12485.

9. Hoffmaster AR, AuCoin D, Baccam P, Baggett HC, Baird R, Bhengsri S, Blaney DD, Brett PJ, Brooks TJ, Brown KA, 2015. Melioidosis diagnostic workshop, 2013. Emerg Infect Dis 21: e141045.

10. Amornchai $P$ et al., 2007. Accuracy of Burkholderia pseudomallei identification using the API 20NE system and a latex agglutination test. J Clin Microbiol 45: 3774-3776.

11. Weissert C, Dollenmaier G, Rafeiner P, Riehm J, Schultze D, 2009. Burkholderia pseudomallei misidentified by automated system. Emerg Infect Dis 15: 1799-1801.

12. Lau SKP, Tang BSF, Curreem SOT, Chan T-M, Martelli $P$, Tse CWS, Wu AKL, Yuen K-Y, Woo PCY, 2012. Matrix-assisted laser desorption ionization-time of flight mass spectrometry for rapid identification of Burkholderia pseudomallei: importance of expanding databases with pathogens endemic to different localities. J Clin Microbiol 50: 3142-3143.

13. Coalition for African Rice Development, 2016. Rice Production in Sub-Sahara Africa. Available at: http://riceforafrica.net/ index.php/production. Accessed August 8, 2016.

14. Carugati M et al., 2018. Incidence of human brucellosis in the Kilimanjaro region of Tanzania in the periods 2007-2008 and 2012-2014. Trans $R$ Soc Trop Med Hyg 112: 136-143.

15. Maze MJ et al., 2016. Comparison of the estimated incidence of acute leptospirosis in the Kilimanjaro region of Tanzania between 2007-08 and 2012-14. PLoS Negl Trop Dis 10: e0005165.

16. Anuntagool N, Naigowit $P$, Petkanchanapong V, Aramsri $P$, Panichakul T, Sirisinha S, 2000. Monoclonal antibody-based rapid identification of Burkholderia pseudomallei in blood culture fluid from patients with community-acquired septicaemia. J Med Microbiol 49: 1075-1078.

17. Alexander AD, Huxsoll DL, Warner AR, Jr., Shepler V, Dorsey A, 1970. Serological diagnosis of human melioidosis with indirect hemagglutination and complement fixation tests. Appl Microbiol 20: 825-833.

18. US CDC, 2012. Melioidosis (Burkholderia Pseudomallei) 2012 Case Definition. Available at: https://wwwn.cdc.gov/nndss/ conditions/melioidosis/case-definition/2012/. Accessed May 23, 2019.

19. Armstrong PK, Anstey N, Kelly P, Currie B, Martins N, Dasari P, Krause V, 2005. Seroprevalence of Burkholderia pseudomallei in East Timorese refugees: implications for healthcare in East Timor. Southeast Asian J Trop Med Public Health 36: 1496-1502.

20. Tiyawisutsri R, Peacock SJ, Langa S, LimmathurotsakulD, Cheng AC, Chierakul W, Chaowagul W, Day NP, Wuthiekanun V, 2005. Antibodies from patients with melioidosis recognize Burkholderia mallei but not Burkholderia thailandensis antigens in the indirect hemagglutination assay. J Clin Microbiol 43: 4872-4874.

21. Steinmetz I et al., 2018. Melioidosis in Africa: time to uncover the true disease load. Trop Med Infect Dis 3: 62.

22. Frazer DN, 1982. Melioidosis. J R Army Med Corps 128: 123-130.

23. James GL, Delaney B, Ward L, Freeman K, Mayo M, Currie BJ, 2013. Surprisingly low seroprevalence of Burkholderia pseudomallei in exposed healthy adults in the Darwin region of tropical Australia where melioidosis is highly endemic. Clin Vaccine Immunol 20: 759-760.

24. Cheng AC, Wuthiekanun V, Limmathurotsakul D, Chierakul W, Peacock SJ, 2008. Intensity of exposure and incidence of melioidosis in Thai children. Trans $R$ Soc Trop Med Hyg 102: S37-S39.

25. Parameswaran U, Baird RW, Ward LM, Currie BJ, 2012. Melioidosis at Royal Darwin Hospital in the big 2009-2010 wet season: comparison with the preceding 20 years. Med J Aust 196: 345-348.

26. Gilmore G, Barnes J, Ketheesan N, Norton R, 2007. Use of antigens derived from Burkholderia pseudomallei, B. thailandensis, and $B$. cepacia in the indirect hemagglutination assay for melioidosis. Clin Vaccine Immunol 14: 1529-1531.

27. Waag DM, England MJ, DeShazer D, 2012. Humoral immune responses in a human case of glanders. Clin Vaccine Immunol 19: 814-816.

28. Wiersinga WJ, Birnie E, Weehuizen TA, Alabi AS, Huson MA, Huis $R A, 2015$. Clinical, environmental, and serologic surveillance studies of melioidosis in Gabon, 2012-2013. Emerg Infect Dis 21: 40-47.

29. Limmathurotsakul D et al., 2013. Systematic review and consensus guidelines for environmental sampling of Burkholderia pseudomallei. PLoS Negl Trop Dis 7: e2105.

30. Scheer CS, Fuchs C, Gründling M, Vollmer M, Bast J, Bohnert JA, Zimmermann K, Hahnenkamp K, Rehberg S, Kuhn S-O, 2019. Impact of antibiotic administration on blood culture positivity at the beginning of sepsis: a prospective clinical cohort study. Clin Microbiol Infect 25: 326-331. 